\title{
STEM Aberration Correction: Where Next?
}

\author{
O.L. Krivanek, N. Dellby, M. Murfitt, P.D. Nellist and Z. Szilagyi
}

Nion Co., $11028^{\text {th }}$ St., Kirkland, WA 98033, USA

Present-day aberration-corrected scanning transmission electron microscopes (STEMs) improve the attainable resolution at a given operating voltage by 2-3x compared to their uncorrected cousins [1]. There are now four such instruments in laboratories in North America. Sub-Angström probe size has become available even at 100-120 keV operating energies [2], and the current available in the ultra-small probe has risen considerably compared to uncorrected instruments. In this paper we summarize some of these recent achievements and look ahead at expected future developments.

Depending on the complexity and the precision of the corrector, the probe shape produced by the STEM depends on 25 or more different aberration coefficients. Further influential factors are the chromatic aberration and energy spread, the geometrical size of the projected electron source, the size, position and cleanliness of the beam-defining aperture and the mechanical and electrical stabilities of the whole instrument. Successfully managing this complexity was one of the key factors that made practical aberration correction possible. Recent modifications of our Ronchigrambased autotuning software [3] have enabled it to determine all the aberration coefficients up to $5^{\text {th }}$ order ( 25 coefficients total) in about 20 seconds, and improved its precision.

As an example of performance now routinely possible, Fig. 1 shows unprocessed high angle annular dark field (HAADF) images of gold particles and single gold atoms on a carbon film recorded with our second generation quadrupole-octupole corrector [1] at $100 \mathrm{kV}$. Even though the supporting carbon film is over $10 \mathrm{~nm}$ thick, single Au atoms can be readily distinguished once the contrast is suitably expanded (Fig. $1 \mathrm{~b}$ and c). In such images, atomic rafts one monolayer thick which connect the particles become readily apparent. Unlike the particles which are fairly stationary, the rafts and the single atoms are highly mobile, facilitating continuous transport of matter from particle to particle. Profiles taken through images of the single atoms and the rafts show that the image levels are quantized, and that the single atom images are tail-free and typically 1.0 to $1.5 \AA$ wide.

The most important remaining limits on the performance of the corrected STEMs arise from a) higher order (fifth and above) aberrations, b) chromatic aberration, and c) the overall stability of the whole electron microscope. We are addressing them as follows:

a) The resolution limits due to fifth and seventh order aberrations are given by:

$$
\mathrm{d}_{5}=0.4 \mathrm{C}_{5}{ }^{1 / 6} \lambda^{5 / 6} \quad \text { and } \quad \mathrm{d}_{7}=0.4 \mathrm{C}_{7}{ }^{1 / 8} \lambda^{7 / 8} \text {. }
$$

Our present $\left(2^{\text {nd }}\right.$ generation) corrector nulls aberrations up to $4^{\text {th }}$ order but produces $\mathrm{C}_{5}$ of about 10 $\mathrm{cm}$, which gives a resolution limit of $0.8 \AA$ at $100 \mathrm{kV}$. At $300 \mathrm{kV}$, however, $\mathrm{d}_{5}$ is lowered to less than $0.5 \AA$ for the same corrector. We are now building a second generation $C_{s}$ corrector for the 300 $\mathrm{kV}$ VG HB603 [4]. More radically, our $3^{\text {rd }}$ generation corrector [5] will null all geometric aberrations up to $6^{\text {th }}$ order, and will give $C_{7}$ of about $20 \mathrm{~cm}$. The resolution limit due to geometric aberrations with this system will be just $0.3 \AA$ even at $100 \mathrm{keV}$.

b) The principal effect of chromatic aberration on STEM HAADF images is to transfer intensity from the probe center into the probe tail, without greatly affecting the shape of the central maximum [1]. This is best described by the fraction $\mathrm{f}_{\mathrm{s}}$ of the total electron flux that is shifted away from the central maximum:

$$
\mathrm{f}_{\mathrm{s}}=(1-\mathrm{w})^{2}, \quad \text { where } \mathrm{w}=2 \mathrm{~d}_{\mathrm{g}}{ }^{2} \mathrm{E}_{\mathrm{o}} /\left(? \mathrm{E} \mathrm{C}_{\mathrm{c}} \lambda\right) \text { or } \mathrm{w}=1 \text {, whichever is smaller. }
$$


$\mathrm{d}_{\mathrm{g}}$ is the resolution in the absence of chromatic aberration. At a resolution $\mathrm{d}_{\mathrm{g}}=0.8 \AA$, energy spread $? \mathrm{E}=0.5 \mathrm{eV}$, coefficient of chromatic aberration $\mathrm{C}_{\mathrm{c}}=1.5 \mathrm{~mm}$ and primary energy $\mathrm{E}_{\mathrm{o}}=100 \mathrm{keV}$, the above gives $\mathrm{f}_{\mathrm{s}}=30 \%$ as the fraction of the electron flux shifted out of the probe maximum into the probe tail. This shows that with the low energy spread of a cold field emission gun, the present-day $100 \mathrm{kV}$ performance is not strongly limited by chromatic aberration. At higher primary energies the influence of chromatic aberration on HAADF imaging willbe even less.

c) Overall stability of the whole instrument becomes increasingly important as the electron-optical resolution is improved to $1 \AA$ and beyond. Present-day electron microscopes typically use columns that were designed several decades ago when 2-3 $\AA$ was the best resolution expected. In order to reach sub- $\AA$ resolution, they tend to use incremental fixes that fall short of the required overall performance. We are addressing this problem by developing a whole new STEM column, specifically designed for sub- $\AA$ stability even in mildly problematic operating environments.

In summary, aberration-corrected STEM has progressed from a curiosity to an accepted scientific instrument. The performance of the corrected STEMs is now limited by obstacles other than spherical aberration. Overcoming the obstacles will likely be accomplished in the next few years. Beyond them lies the ultimate resolution limit - the size of an atom - and an era of electron microscopy in which limitations due to electron optics will no longer be the major concern.

\section{References}

[1] N. Dellby et al., J. Electron Microscopy 50 (2001) 177.

[2] P.E. Batson et al., these proceedings.

[3] O.L. Krivanek et al., US patent application (2000).

[4] S.J. Pennycook et al., these proceedings.

[5] O.L. Krivanek et al., to be published.
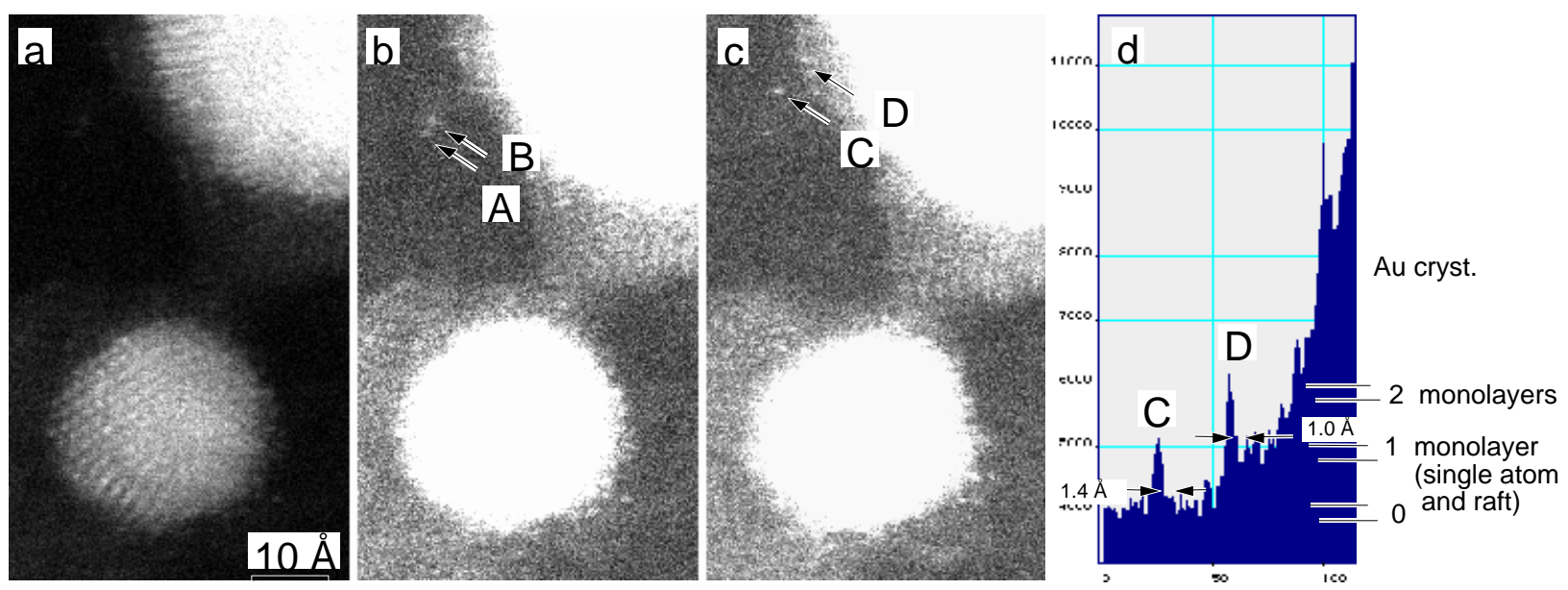

Fig. 1. Unprocessed HAADF images of gold particles, monolayer rafts and single Au atoms. 32 msec per pixel, pixels $0.18 \AA$ wide. a) HAADF image, b) same as (a) but with boosted contrast, c) HAADF image taken under the same conditions as (b) but 100 seconds later, d) line profile through atoms $\mathrm{C}$ and $\mathrm{D}$ plus adjacent raft and crystal in image (c). Note that atoms $\mathrm{A}$ and $\mathrm{B}$ have moved elsewhere during the 100 seconds and that the image of the single atom D sitting at the edge of a monolayer raft is just $1.0 \AA$ wide. 